\title{
Separation of Granules Containing Melanophore Stimulating Hormone from Frog (Rana Catesbeiana) Pars Intermedia
}

\author{
SAKAÉ KIKUYAMA AND IKUO YASUMASU \\ Department of Biology, School of Education, Waseda University, Tokyo
}

\begin{abstract}
Synopsis
Granules containing melanophore stimulating hormone were separated from bullfrog pars intermedia by differential centrifugation and sucrose gradient centrifugation. These granules were present in the supernatant after centrifugation at $650 \mathrm{~g}$ for $15 \mathrm{~min}$ and were sedimented by centrifugation at $11,000 \mathrm{~g}$ for $15 \mathrm{~min}$. Sucrose gradient centrifugation of this fraction revealed that $\mathrm{MSH}$ activity was present in granules having a density equivalent to $1.6-1.7 M$ sucrose solution and a diameter $1,500-3,000 \AA$. From their size and structure, it is concluded that MSH granules are the small electron-dense granules and not the large colloidal granules observed in electron micrographs of the pars intermedia.
\end{abstract}

Recently, the fine structure of the intermediate lobe of the pituitary has been studied in amphibians (Iturriza, 1964; Imai and Oota, 1965; Cohen, 1967; Pehlemann, 1967; Saland, 1968; Ito, 1968). It was reported that there exist two types of granules; relatively small vesicles and large droplets (in Bufo arenarum: Iturriza, 1964; Xenopus leveis: Imai and Oota, 1965; Rana pipiens and Rana catesbeiana: Saland, 1968). The present authors have also observed both types of granules in the intermediate lobe of Rana catesbeiana and Rana nigromaculata.

To our knowledge, no report exists which provides a definite evidence that either or both of these two types of granules contain melanophore stimulating hormone (MSH).

In the present experiments, attempts were made to separate a fraction having potent MSH activity and to determine which type of granules contains MSH.

A portion of this work has been reported in

Received for publication October 25, 1972. abstract form (Kikuyama and Yasumasu, 1969).

\section{Materials and Methods}

Obtaining glands:

Bullfrog (Rana catesbeiana) pituitaries were dissected from the head region after decapitation. The pars intermedio-posterior was freed from the pars anterior. Procedures were carried out in a cold room at $2-4{ }^{\circ} \mathrm{C}$.

Preparation of pituitary homogenate:

$520-760 \mathrm{mg}$ of tissue (about $150-200$ pieces) were processed at one time. A $10 \%$ homogenate of the tissue was prepared in $0.25 \mathrm{M}$ sucrose solution.

\section{Centrifugation:}

For differential centrifugation, a refrigerated centrifuge (90 UV, Tominaga) and ultra-centrifuge (55P, Hitachi) were used. The latter centrifuge was also employed for sucrose gradient centrifugation. The procedures are described in the following section.

Maesurement of amounts of protein in fractions:

Each particulate fraction obtained was suspended in $2-10 \mathrm{~m} l$ of $0.25 M$ sucrose. Fractions obtained by sucrose gradient centrifugation were diluted with 
distilled water to $0.25 \mathrm{M}$, and then their volumes were adjusted to $10 \mathrm{~m} l$ with $0.25 M$ sucrose. Onetenth mililiter of each suspension was used for protein assay by the method of Lowry et al. (1951).

\section{Morphological examination of fractions:}

Pellets were obtained by centrifugation $(30,000 \mathrm{~g}$, $30 \mathrm{~min}$ ) of one half of the volume of the suspension of the fractions. They were fixed in $2 \%$ glutaraldehyde in $0.05 M$ cacodylate buffer for $2 \mathrm{hr}$, post-fixed in $1 \%$ osmic acid in $0.05 M$ Veronal buffer for $1 \mathrm{hr}$ and embedded in Maraglas D.E.R.-732 after passing through an alcohol series. Thin sections were taken from several parts of each pellet and were stained with lead citrate (Reynolds, 1963) for observation with electron microscope (JEM-17).

\section{Determination of fraction having the highest MSH activity}

One $\mathrm{m} l$ of the suspension of each fraction was mixed with $1 \mathrm{~m} l$ of $2 N$ acetic acid. After $1 \mathrm{hr}$ at room temperature, the suspension was centrifuged at $30,000 \mathrm{~g}$ for $30 \mathrm{~min}$. The suspension freed of precipitate was neutralized with $1 \mathrm{~N}$ sodium hydroxide and stored in the cold until assayed. The extracts of each fraction were diluted 10 times in a stepwise fashion with a mixture of 3 parts of Gey's solution (1949) and 1 part of distilled water. The skin of the ventral region of a premetamorphic bullfrog tadpole which had been adapted on a white background for a few days was dissected off into the solution. The skin was cut into pieces about $4 \mathrm{~mm}$ square. One piece was immersed in $1 \mathrm{~m} l$ of each dilution of each fraction contained in a test tube. After $1 \mathrm{hr}$, the pieces were taken out, placed on a glass slide and observed under a light microscope for reading melamophore index (Landgrebe and Waring, 1944). The highest dilution which gave melanophore index of $3\left(D_{3}\right)$ was obtained in each fraction. The fraction which showed the highest $\mathrm{D}_{3}$ value was regarded as the one having the highest MSH activity. The test was repeated 2-3 times. All the extracts of fractions obtained in a series of experiments were assayed with the skin pieces from one specimen to avoid errors due to the individual differences of sensitivity of the skin to MSH. Sensitivity of the skin was determined

Table 1. Distribution of MSH activity in fractions obtained by differential centrifugation, Expriment A

\begin{tabular}{llllrr}
\hline Fraction & I & II & III & IV & \multicolumn{1}{c}{ V } \\
\hline $\log \mathrm{D}_{3}$ & 2 & $2-3$ & $3-4$ & 5 & 2 \\
Protein $(\%)$ & 8 & 11 & 19 & 33 & 29 \\
\hline
\end{tabular}

by testing the pieces in a series of standard solution $(0.01 \mathrm{IU} / \mathrm{m} l$ ACTH, Scherring). The skin pieces were used for the assay only when they exhibited melanophore index of 5, 3 and 1 in the 1, 10 and 100 times dilutions of the standard solution respectively.

\section{Experiments and Results}

\section{Differential centrifugation.}

The homogenate of pars intermedio-posterior was fractionated by several methods of differential contrifugation.

\section{Experiment $A$ :}

Fraction I was the sediment obtained by centrifugation at $27 \mathrm{~g}$ for $15 \mathrm{~min}$, Fraction II, at $650 \mathrm{~g}$ for $15 \mathrm{~min}$, Fraction III, at $1,700 \mathrm{~g}$ for $15 \mathrm{~min}$ and Fraction IV, at $11,000 \mathrm{~g}$ for $15 \mathrm{~min}$. Fraction $\mathrm{V}$ was the supernatant after the last centrifugation. As shown in Table 1, MSH activity was located mainly in Fraction IV.

\section{Experiment $B$ :}

Six fractions were obtained by the following procedure: Fraction I was the precipitate following centrifugation at $110 \mathrm{~g}$ for $15 \mathrm{~min}$, Fraction II, at $650 \mathrm{~g}$ for $15 \mathrm{~min}$. Fraction III, at $3,700 \mathrm{~g}$ for $15 \mathrm{~min}$, Fraction IV, at $30,000 \mathrm{~g}$ for $30 \mathrm{~min}$ and Fraction V, at $105,000 \mathrm{~g}$ for $60 \mathrm{~min}$. The supernatant remaining after the last centrifugation was designated as Fraction VI. Assays for MSH revealed that the activity was located largely in Fraction III (Table 2).

\section{Experiment $C$ :}

Fraction I was the precipitate obtained

Table 2. Distribution of MSH activity in fractions obtained by differential centrifugation, Experiment B

\begin{tabular}{llrllll}
\hline Fraction & I & II & III & IV & V & VI \\
\hline Log $\mathrm{D}_{3}$ & $2-3$ & 3 & $4-5$ & $3-4$ & 2 & 3 \\
Protein $(\%)$ & 11 & 17 & 29 & 27 & 7 & 9 \\
\hline
\end{tabular}


Table 3. Distribution of MSH activity in fractions obtained by differential centrifugation, Experiment $\mathrm{C}$

\begin{tabular}{lllrrrl}
\hline Fraction & I & II & III & IV & V & VI \\
\hline Log $\mathrm{D}_{3}$ & $2-3$ & $3-4$ & 5 & 3 & 2 & 3 \\
Protein $(\%)$ & 11 & 25 & 35 & 13 & 7 & 9
\end{tabular}

following centrifugation at $110 \mathrm{~g}$ for $15 \mathrm{~min}$, Fraction II, at $1,700 \mathrm{~g}$ for $15 \mathrm{~min}$, Fraction III, at $11,000 \mathrm{~g}$ for $15 \mathrm{~min}$, Fraction IV, at $30,000 \mathrm{~g}$. for $30 \mathrm{~min}$ and Fraction $\mathrm{V}$, at $105,000 \mathrm{~g}$ for $60 \mathrm{~min}$. Fraction VI was obtained as the supernatant of the last centrifugation. MSH activity was located in Fraction III (Table 3).

\section{Experiment $D$ :}

Fraction I was the sediment following centrifugation at $650 \mathrm{~g}$ for $15 \mathrm{~min}$, Fraction II, at $11,000 \mathrm{~g}$ for $15 \mathrm{~min}$, Fraction III, at $30,000 \mathrm{~g}$ for $30 \mathrm{~min}$ and Fraction IV, at 105,000 $\mathrm{g}$ for $60 \mathrm{~min}$. Fraction $\mathrm{V}$ was the supernatant of the last centrifugation. As shown in Table 4, MSH activity was located largely in Fraction II which represents each fraction having the highest MSH activity obtained by Experiment A, B and C. Electron-microscopical examination indicated that Fraction I contained large electron dense granules (Fig. 1) very similar in size and structure to the large granules found in the intact intermediate lobe (Fig. 2 ), in addition to nuclei, fragments of fibrous tissue and nerve endings. Fraction II contained nerve endings, mitochondria and electron dense granules (Fig. 3), most of which were similar to the small granules seen in the intermediate lobe (Fig. 4). Fraction III was composed of electron dense granules (Fig. 5) which were smaller than most of the granules in Fraction II. They resembled the granules in the nerve endings. In Fraction III, small electron lucent granules were also present. Fraction IV contained several types of minute particles.
Table 4. Distribution of MSH activity in fractions obtained by differential centrifugation, Experiment D

\begin{tabular}{lrrllr}
\hline \hline Fraction & I & II & III & IV & V \\
\hline Log $\mathrm{D}_{3}$ & 3 & 5 & $3-4$ & 2 & 3 \\
Protein $(\%)$ & 17 & 38 & 12 & 9 & 24 \\
\hline
\end{tabular}

Table 5. Distribution of MSH activity in fractions prepared by sucrose gradient centrifugation of Fraction II obtained by differential centrifugation, Experiment D

\begin{tabular}{lllrlr}
\hline Fraction & I & II & III & IV & \multicolumn{1}{c}{ V } \\
\hline $\log \mathrm{D}_{3}$ & $2-3$ & $2-3$ & 3 & $4-5$ & 3 \\
Protein $(\%)$ & 11 & 17 & 16 & 40 & 16 \\
\hline
\end{tabular}

\section{Sucrose gradient centrifugarion.}

Sucrose gradient centrifugation was carried out to determine whether there are $\mathrm{MSH}$ containing particles of a particular density in Fraction II obtained by differential centrifugation, Experiment D. A sucrose gradient (1-2 $M)$ was prepared in three plastic tubes $(5 \mathrm{ml})$. Over the top sucrose layer of each tube, $0.5 \mathrm{~m} l$ of the $0.5 \mathrm{M}$ sucrose suspension of Fraction II was layered. Equilibrium of the system was obtained by centrifugation at 40,000 r.p.m. for $3 \mathrm{hr}$ in swing rotor (RSP-40). At the termination of centrifugation, the content of each tube was divided into 5 fractions using a tube slicer. In every tube, 4 opaque bands were invariably observed (Fig. 6). Fractions from 2 of the 3 tubes were used for MSH assay and for measurement of the amount of protein. MSH activity was largely present in Fraction IV (1.6-1.7 $M$ sucrose) (Table 5). Of the 5 fractions in the remaining tube, only 2 fractions (Fractions III and IV) yielded enough precipitate for fixation by centrifugation carried out after each fraction was diluted to $0.25 M$. Electron microscopical study revealed that in Fraction III, mitochondria, nerve endings and fragments of membranous structures were present (Fig. 7). Fraction IV was mainly composed of electron dense granules 

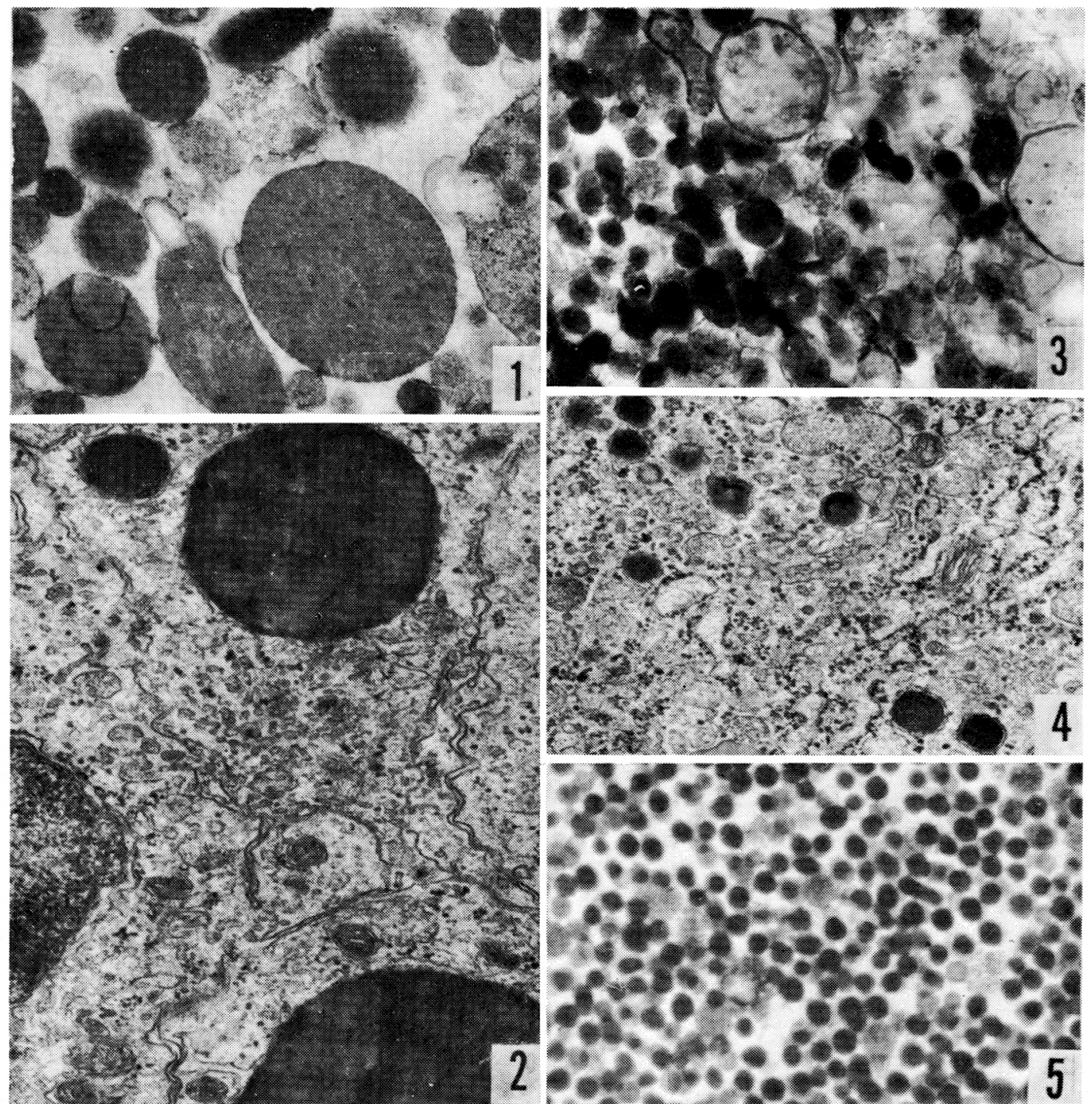

Fig. 1. Electronmicrograph of Fraction I obtained by differential centrifugation of pars intermedio-posterior homogenate, Experiment D. $\times 20,000$.

Fig. 2. Electronmicrograph of intact pars intermedia of a bullfrog. Note large electron dense granules. $\times 20,000$.

Fig. 3. Electronmicrograph of Fraction II obtained by differential centrifugation of pars intermedio-posterior homogenate, Experiment D. $\times 20,000$.

Fig. 4. Electronmicrograph of pars intermedia of a bullfrog. Note membrane-bound small secretory granules. $\times 20,000$.

Fig. 5. Electronmicrograph of Fraction III obtained by differential centrifugation of pars intermedio-posterior homogenate, Experiment D. $\times 20,000$. 


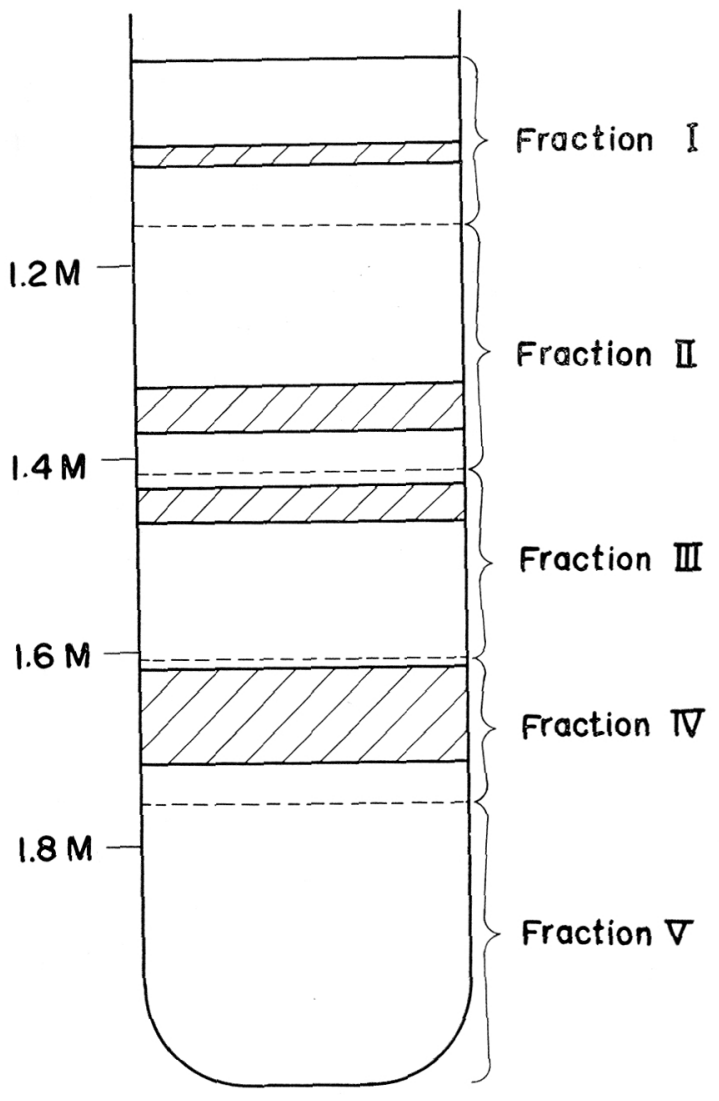

Fig. 6. Fractionation by sucrose gradient centrifugation of Fraction II obtained by differential centrifugation, Experiment D. Diagonal line zones indicate opaque bands observed in a tube after centrifugation.

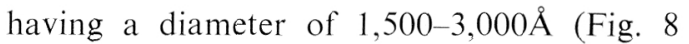
and 9).

\section{Discussion}

In the present experiment, we used the complex of partes nervosa and intermedia as a starting meterial since they were inseparable By the electron microscopical observation of the intact tissue of the pars intermedioposterior, we noticed that the intermediate lobe cells contained more or less irregularshaped small granules and that the nerve endings in the posterior lobe contained the round-shaped secretory granules. However, once they were fractionated it became difficult to discriminate between the small granules of the intermediate lobe origin and the neurosecretory granules of the posterior lobe. According to Oota and Kobayashi (1963), the diameter of neurosecretory granules in the pars nervosa of Rana catesbeiana was 530$1950 \AA$. In the present experiment, the granules in the fraction which exhibited high MSH

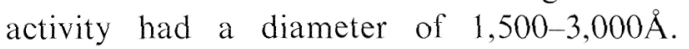
Therefore, it is probable that a part of granules in Fraction IV obtained by density gradient centrifugation are of neurohypophyseal origin. The fact that too much protein (about $15 \%$ of the total protein in the starting
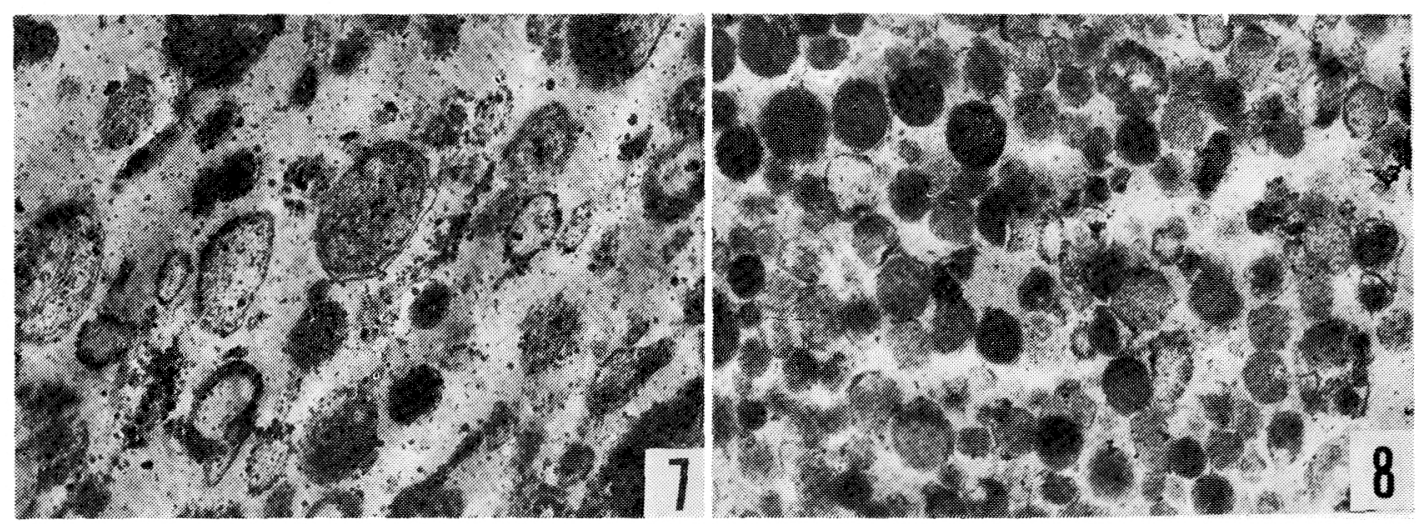

Fig. 7. Electronmicrograph of Fraction III obtained by sucrose gradient centrifugation of Fraction II separated by differential centrifugation, Experiment D. $\times 20,000$.

Fig. 8. Electronmicrograph of Fraction IV obtained by sucrose gradient centrifugation of Fraction II separated by differential centrifugation, Experiment D. $\times 20,000$. 


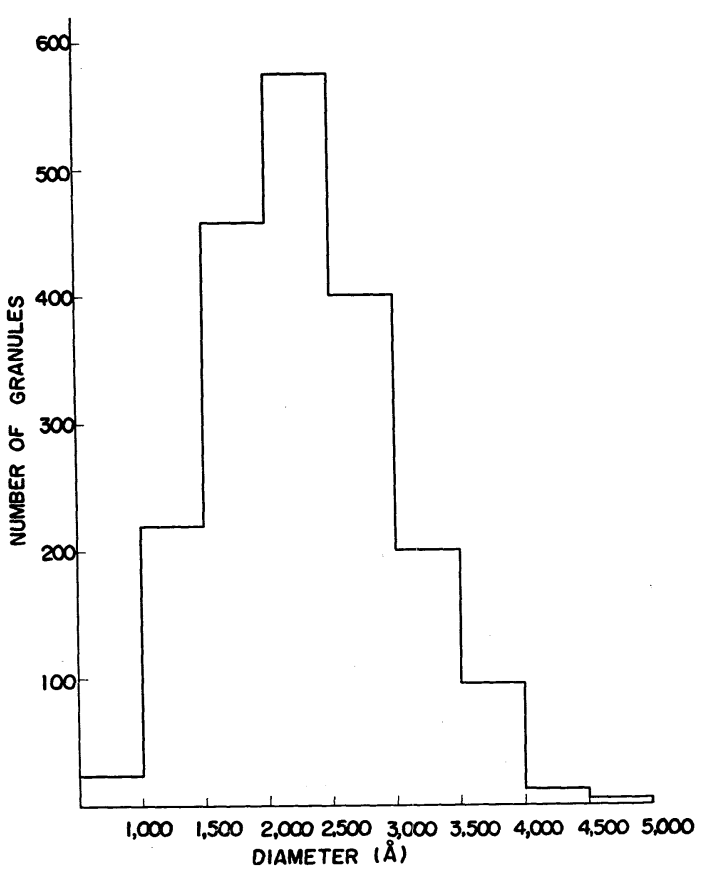

Fig. 9. Distribution of granules in Fraction IV obtained by sucrose gradient centrifugation. Two thousand granules were measured.

material) was recovered in Fraction IV also suggests that this fraction is not composed of MSH granules only. However, the presence of strong MSH activity indicates that the most of the MSH granules are present in this fraction. Therefore, it is concluded that the MSHcontaining granules are the small secretory granules in the pars intermedia.

It has been reported that the population of these granules in the pars intermedia of Rana pipiens and Rana catesbeiana was fairly constant under the varying condition (Saland, 1968). Ito (1968) observed in the pars intermedia of Rana nigromaculata that similar granules decreased in number after the hypothalamo-hypophysial tract was transected.

The population of large colloidal granules in the intermediate lobe of frogs adapted to white or black backgrounds seems to vary among the species studied. The intermediate lobe from black-adapted bullfrogs contains many large droplets; these droplets were seen infrequently or not at all in pars intermedia of black-adapted Rana pipiens (Saland, 1968). According to Imai (1971), these droplets were more frequently observed in the intermediate lobe from black-adapted frogs, Хenopus laevis, than in the gland from white-adapted animals.

Iturriza (1964) observed in the pars intermedia of Bufo arenarum that the large droplets were in close contact with the endings of nonneurosecretory nerves. He assumed that the masses are representative of stored MSH and that the nerves in contact with the masses are controlling the storage of the hormone. In the present experiments, however, the fraction which contained large electron dense granules similar in characteristics to the colloidal masses observed by Iturriza did not exhibit strong MSH activity. This strongly suggests that large granules do not contain $\mathrm{MSH}$, at least in an active form. Imai (1971) also claimed that the colloidal substance is not a reservoir of $\mathrm{MSH}$, since the MSH content is higher in white-adapted Xenopus leavis in which the colloidal substance is absent than in blackadapted ones where the substance is abundant.

In the present experiments, MSH activity of the supernatant fraction after the centrifugation at $105,000 \mathrm{~g}$ was much lower than expected. In the MSH granules, there may be a certain protein for which MSH has a strong affinity as suggested by Namiki et al. (1969).

\section{References}

Cohen, A. G. (1967). Nature 215, 55.

Gey, G. O. (1949). Bulletin of the tissue culture association March 16.

Imai, K. Biology of Normal and Abnormal Melanocytes, edited by T. Kawamura, T. B. Fitspatrick and M. Seiji, University of Tokyo Press, p. 17, (1971).

Imai, K. and Y. Oota (1965). Jap. J. Exptl. Morphol. 19, 89. (in Japanese).

Ito, T. (1968). Neuroendocrinology 3, 35. 
Iturriza, F. C. (1964). Gen. Comp. Endocrinol. $6,19$.

Kikuyama, S. and I. Yasumasu (1969). Zool. Mag. 78, 22. (in Japanese).

Landgrebe, F. W. and H. Waring (1944). Quart. J. Exptl. Physiol. 33, 1.

Lowry, O. H., N. J. Rosebrough, A. L. Farr and R. J. Randall (1951). J. Biol. Chem. 193, 265.
Namiki, H., S. Kikuyama and I. Yasumasu (1969). Zool. Mag. 78, 23. (in Japanese).

Oota, Y. and H. Kobayashi (1963). Z. Zellforsh. 60, 685.

Pehlemann, F. W. (1967) Gen. Comp. Endocrinol. 9, 481.

Reynolds, E. S. (1963). J. Cell Biol. 17, 208.

Saland, L. C. (1968). Neuroendocrinology 3, 72. 